\title{
Application of RT-PCR in SARS-CoV-2 Diagnostics: The Lab Scientist Perspective
}

\author{
Murugan $\mathrm{N}^{1 *}$, Deepa $\mathbf{M}^{1}$, Chirayu $\mathbf{P}^{1}$ and Premina $\mathrm{S}^{2}$ \\ ${ }^{1}$ Molecular Testing-Infectious Disease division, Life Cell International Pvt Ltd, India \\ ${ }^{2}$ Department of Microbiology, Madras Christian College, India
}

*Corresponding author: Dr. Murugan Nandagopal, Scientists \& Deputy General Manager, Molecular Genetics-Infectious Diseases (ID) Division, R\&D, Life Cell International Pvt Ltd, Vandalur Kelambakkam Main Road, Keelakottaiyur, Chennai 600048, Tamil Nadu, India, Email: murugan2science@gmail.com

Keywords: COVID 19; RT PCR; Applications; Diagnosis

Abbreviations: SARS CoV-2: Severe Acute Respiratory Syndrome Coronavirus 2; NGS: Next Generation Sequencing; NAAT: Nucleic Acid Amplification Tests; RdRp: RNA dependent RNA polymerase; Orf1ab: Open Reading Frame 1 A \& B; CT: Computed Tomography; LOD: Limit of Detection.

\section{Introduction}

Severe acute respiratory syndrome coronavirus 2 (SARS CoV-2) was identified and reported from Wuhan, China during the month of December 2019 by a group of scientist. Initially, it was suspected to be a common cold virus causing wide group of community being affected by Pneumoniae and admitted for severe respiratory syndrome. Instant, spike of mortality and morbidity were observed, which leads to the suspicious on the epidemiology of the disease. Zhu $\mathrm{N}$, et al. [1] discovered that the novel Coronavirus causing Pneumonia tends to be severe respiratory diseases using Next Generation sequencing (NGS) technologies, scientist isolated the virus from clinical specimen and sequenced through NGS method and found to be a novel Virus with $82 \%$ homology of known SARS-CoV-1, hence the WHO designated the novel Coronavirus as SARS-CoV-2.

Though NGS, were used to identify the novel Coronavirus, which is expensive and time consuming, which may not be applied for the diagnosis of this pandemic situation. Hence, highly sensitive, specific with target genome sequence using TaqMan probes were designed by various manufactures around the globe. In this communication, we tried to elaborate the applications, advantages and pitfalls of using RT PCR in SARS-CoV-2 diagnosis with our diagnostic experiences gained during this period of May 2020 to Feb 2021 and other literature based survey.

\section{Background}

Globally, lockdown made the human under extensive pressure, irrespective of age, gender, socio-economic status. The only positive approach was the diagnosis of SARS-CoV-2 among the community at short time with higher sensitivity. In the beginning of February 2020, RT-PCR kits were not available in the market for instant diagnosis, due to the Pandemic situation and the requirements were larger over the globe. Later on, Global market turned on RT PCR Kit development against SARS-CoV-2 diagnosis, trend to achieve the diagnosis and prognosis development.

Until, this Pandemic situation, RT-PCR test/Nucleic acid amplification tests (NAAT) was not applied widely for Infectious disease diagnosis, but the whole scenario changed upon the arrival of COVID-19. The RT-PCR test became the backbone of diagnostics in COVID-19 because of their sensitivity and feasibility as compared to the viral culture and NGS techniques. Since, the conventional PCR is not sensitive as TaqMan probe based RT PCR open system; it was widely accepted by the scientific and diagnostic community around the globe [2].

\section{Applications of RT PCR in SARS-CoV-2 Diagnosis}

A variety of RT-PCR assay was developed during the pandemic SARS-CoV-2 including a commercial ready to use RT-PCR kit and has become a gold standard method for the 
detection of nucleic acid [2,3]. All over the globe developed various commercially available kits for detecting the SARSCoV-2 targeting various preliminary and confirmatory genes. Envelope (E) gene, Spike (S) gene, Nucleocapsid (N) gene which are homology to all types of Coronavirus family hence, it was considered as preliminary diagnosis if any one or all three genes found to be positive among any clinical swabs, whereas, Open reading Frame 1 A \& B (Orf1ab) and RNA dependent RNA Polymerase (RdRp) gene was considered as confirmatory gene for the diagnosis of SARS-CoV-2 among various commercial kits Figures 1-2. Most of the kits targeted probe against Orf1ab gene, which consist of wide range of genes involved in genes encoding non-structural protein synthesis. Whereas, RdRp gene is highly specific and narrow range of gene among the SARS-CoV-2 genome, which gives the confirmatory detection system. Few kits targeting $\mathrm{N} 1$ and $\mathrm{N} 2$ gene as a confirmatory genes, which is not appreciated for diagnostic purposes.
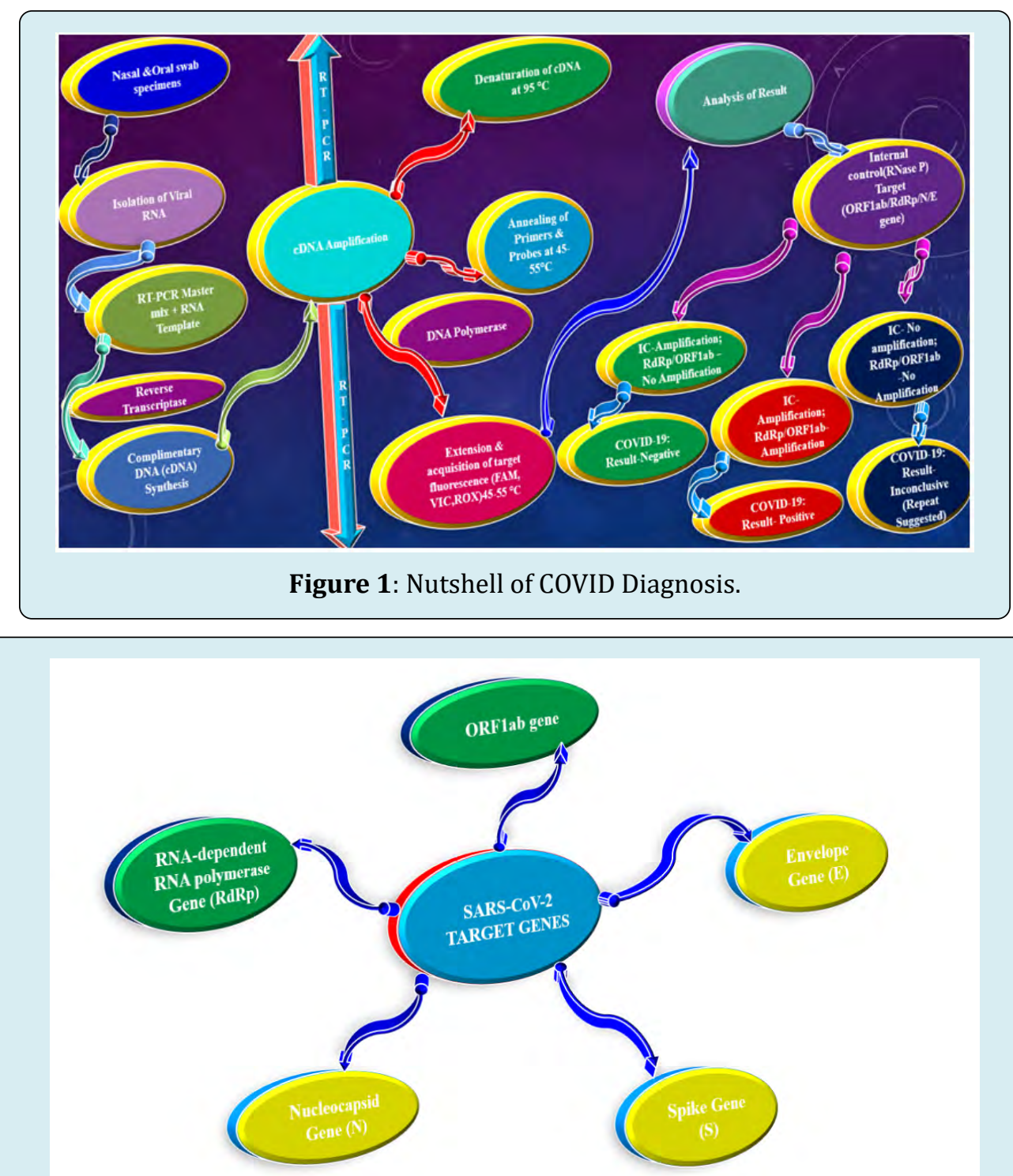

Figure 2: SARS-CoV-2 List of Target genes used for RT-PCR Diagnostic kits. Highlighted in green boxes are confirmatory genes and Yellow were Preliminary detection genes.

\section{Pitfalls of RT PCR in SARS-CoV-2 Diagnosis}

The RT-PCR test has a specificity rate of nearly $100 \%$ among asymptomatic patients but not $100 \%$ among symptomatic patients. We encountered, many patients with
Computed Tomography (CT) scan report shown, bilateral lobe ground glass opacities suggestive of COVID-19, but found to be RT-PCR negative for SARS-CoV-2 test. This could be due to various factors, poor sample collection due to noncooperative patients during the sample collection or the 


\section{Epidemiology International Journal}

untrained paramedic, hence both nasal and oral swabs were encouraged for sample collection to avoid false negative reports. In addition, standard incubation period of SARSCoV-2 is $5-6$ days, sample collected during or the before incubation period may have a higher chance of getting result will be negative because the viral load to the patients is very flat. In lab, there is a chance of poor RNA isolation or amplification process by the untrained staff members. There were many incidences, were the false negative reports were possible, such as poor RNA isolation or sample processing method, error during the master mix reagent preparation, inappropriate thermal profile selection or instrument related issues, improper labelling of samples leading to positive samples reported as negative and vice versa [4]. In another way, positive CT scan report may be due to various other mono/polymicrobial infections that resemble COVID-19 and misdiagnosed as COVID-19 by the clinicians.

A study by Lieberman JA, etal. [5] compared four different commercially available COVID-19 Kits and found all were equally specific and a specific manufacture probe had highly sensitivity at the level of lower limit of Detection (LOD). A study by Kasteren PB, et al. [6] compared seven different commercially available kit and found $96 \%$ efficiency \& $100 \%$ specificity for the identification in the SARS-CoV-19 in low concentration of the clinical samples. Another study by Wang $X$, et al. [7] determined the six LOD RT-PCR kits which was approved by China NMPA for diagnosis in COVID-19 and observed $100 \%$ sensitivity at LOD.

\section{Conclusion}

To conclude, though RT-PCR was expensive and requires dedicated trained person when compared to rapid antigen and antibody test. It is highly sensitive and accurate next to CT scan diagnosis, especially for COVID-19 and chance of false negative is lower compared to other available diagnostic methods. Strict adherence to protocol with appropriate sample collection, RNA isolation, RT PCR processing and report analysis ensures the RT-PCR as gold standard method of COVID-19 diagnosis.

\section{References}

1. Zhu N, Zhang D, Wang W, Li X, Yang B, et al. (2020) A Novel Coronavirus from patients with Pneumonia in China, 2019. N Engl J Med 382: 727-733.

2. Emery SL, Erdman DD, Bowen MD, Newton BR, Winchell JM, et al. (2004) Real-Time Reverse TranscriptionPolymerase Chain Reaction Assay for SARS-associated Coronavirus. Emerg Infect Dis 10(2): 311-316.

3. Holland PM, Abramson RD, Watson R, Gelfand DH (1991) Detection of specific polymerase chain reaction product by utilizing the 5'-3' exonuclease activity of Thermus aquaticus DNA polymerase. Proc Natl Acad Sci 88(16): 7276-7280.

4. Saeed KBA, Ahmad NS (2013) Real-Time Polymerase Chain Reaction: Applications in Diagnostic Microbiology. International Journal of Medical Students 1(1): 28-36.

5. Lieberman JA, Pepper G, Naccache SN, Huang ML, Jerome KR, et al. (2020) Comparison of Commercially Available and Laboratory Developed Assays for in vitro Detection of SARS-CoV-2 in Clinical Laboratories. J Clin Microbiol.

6. van Kasteren $\mathrm{PB}$, van der Veer $\mathrm{B}$, van den Brink $\mathrm{S}$, Wijsman L, de Jonge J, et al. (2020) Comparison of seven commercial RT-PCR diagnostic kits for COVID-19. J Clin Virol 128: 104412.

7. Wang X, Yao H, Xu X, Zhang P, Zhang M, et al. (2020) Limits of Detection of 6 Approved RT-PCR Kits for the Novel SARS-Coronavirus-2 (SARS-CoV-2). Clin Chem 66(7): 977-979. 